\title{
Evaluación de los parámetros quirúrgicos empleando dos plataformas de facoemulsificación en cataratas grado I a IV
}

\section{Surgical parameter assessment using two Phacoemulsification Systems for grade I to IV cataracts}

\author{
Roberto González-Salinas ${ }^{*}$, Claudia Corredor-Ortega², Manuel Garza-León ${ }^{3}$, Marla Álvarez-Padilla², \\ Ana S. Serrano-Ahumada ${ }^{2}$, Fátima Rubio-Tijerina ${ }^{2}$, José A. Martínez-Cano ${ }^{2}$ y \\ Juan P. Olivares-de Emparan ${ }^{2}$
}

${ }^{1}$ Departamento de Investigación, Asociación para Evitar la Ceguera en México IAP, Ciudad de México; ${ }^{2}$ Departamento de Cirugía del Segmento Anterior, Asociación para Evitar la Ceguera en México IAP, Ciudad de México; ${ }^{3}$ División de Ciencias de la Salud, Universidad de Monterrey, Nuevo León. México

\section{Resumen}

Objetivo: Comparar la energía disipada acumulada (CDE), fluido aspirado y tiempo de aspiración utilizado durante la cirugía de catarata por facoemulsificación usando 2 plataformas. Métodos: Se incluyeron los ojos de pacientes consecutivos programados para ser sometidos a cirugía de catarata. Las opacidades del cristalino fueron clasificadas desde NO1NC1 a NO6NC6 utilizando el sistema de clasificación de opacidades del cristalino (LOCS III). Se asignaron 2 plataformas: 1) fluídica activa del Centurion ${ }^{\oplus}$ y 2) fluídica por gravedad de Infiniti ${ }^{\oplus}$. Se registraron y compararon la energía disipada acumulada (CDE), el tiempo de aspiración utilizado y el fluido aspirado estimado. Resultados: Un total de 216 ojos fueron evaluados. La edad media $\pm D E$ fue de $78.3 \pm 9.8$ años (rango 57 a 92). Se observó una diferencia estadísticamente significativa en la CDE para cataratas grado II a IV ( $p=0.031, p=0.045$ y $p=0.032$, respectivamente). El tiempo de aspiración mostró una disparidad significativa para cataratas grado II y III ( $p=0.024$ y $p=0.001$, respectivamente), así como el fluido aspirado estimado ( $p=0.001$ y $p=0.042$, respectivamente). Todas las diferencias favorecieron al grupo con la plataforma de fluídica activa. Conclusiones: El sistema de fluídica activa demostró ser más eficiente que la fluídica por gravedad en facoemulsificación de cataratas grado II a IV.

Palabras clave: Cirugía de catarata. Facoemulsificación. Fluídica activa. Fluídica por gravedad. Parámetros quirúrgicos. Energía disipada acumulada.

\section{Abstract}

Aim: To compare the cumulative dissipated energy (CDE), aspiration fluid and aspiration time (AT) used during phacoemulsification using two surgical platforms. Methods: Consecutive eyes from patients undergoing cataract surgery were included. Lens opacities were graded from N01NC1 to NO6NC6 using the LOCS III classification. Two platforms were evaluated: 1. Centurion ${ }^{\circledast}$ system with active-fluidics. 2. Infiniti vision system with gravity-fluidics. CDE, AT and mean estimated as-

Correspondencia:

*Roberto González-Salinas

Vicente García Torres, 46

Barrio San Lucas, Del. Coyoacán Fecha de recepción: 13-04-2018

C.P. 04030, Ciudad de México, México Fecha de aceptación: 15-10-2018

E-mail: dr.gonzalezsalinas@apec.com.mx DOI: 10.24875/RMO.M18000058 0187-4519/@ 2018 Sociedad Mexicana de Oftalmología. Publicado por Permanyer México. Este es un artículo Open Access bajo la licencia CC BY-NC-ND (http://creativecommons.org/licenses/by-nc-nd/4.0/).

Disponible en internet: 02-01-2019 Rev Mex Oftalmol. 2019;93(1):19-25 www.rmo.com.mx www.rmo.com.mx 
piration fluid (EAF) were registered and compared. Results: $A$ total of 216 eyes were evaluated. Mean age \pm standard deviation was $78.3 \pm 9.8$ years (range 57-92). Significant differences were evidenced for overall CDE per nuclear density for cataracts Grade II to IV ( $p=0.031, p=0.045$ and $p=0.032$ respectively). AT showed a significant disparity for cataracts Graded II and III ( $p=0.024$ and $p=0.001)$, as the $\operatorname{EAF}(p=0.001$ and $p=0.042$ respectively). All differences favoured the active-fluidics configuration group. Conclusions: Active-Fluidics System demonstrated to be more effective than Gravity-fluidics system for cataracts Grade II-IV phacoemulsification.

Key words: Cataract surgery. Phacoemulsification. Active-fluidics. Gravity-fluidics. Surgical parameters. Cumulative dissipated energy.

\section{Introducción}

El aumento de la población mundial, además de una mayor esperanza de vida, impone grandes desafíos para los sistemas de salud actuales debido a un aumento de las patologías relacionadas con la edad, como las cataratas ${ }^{1,2}$. Además, a pesar de los esfuerzos recientes para reducir la acumulación de cataratas existente, esta sigue siendo la principal causa de ceguera en todo el mundo ${ }^{3}$.

Actualmente, la facoemulsificación es la técnica quirúrgica preferida en los países de altos ingresos ${ }^{4,5}$, y ha contribuido significativamente a que la extracción de cataratas sea uno de los procedimientos más seguros y rentables en medicina ${ }^{6,7}$. Hoy en día, las expectativas de los pacientes están en aumento y los cirujanos de catarata pretenden obtener el mejor resultado refractivo postoperatorio posible, utilizando métodos más precisos para el cálculo del poder de los lentes intraoculares (LIO), nuevas tecnologías e innovaciones mejoradas en el diseño de LIO, así como sistemas de facoemulsificación más efectivos ${ }^{8-10}$.

El principio de facoemulsificación se basa en el corte mecánico del núcleo del cristalino utilizando una punta oscilante $^{9}$. Hasta 2005, el ultrasonido longitudinal era la única tecnología disponible; sin embargo, la cantidad de poder de ultrasonido requerida para eliminar una catarata dura con la faco tradicional implicaba un riesgo sustancial para las células endoteliales de la córnea. Sin embargo, la faco torsional produce movimientos laterales de la punta, lo que reduce de manera efectiva la cantidad de ultrasonido utilizada y disminuye el efecto repelente de la punta de faco sobre los fragmentos nucleares ${ }^{8,9}$. El ultrasonido transversal se introdujo recientemente combinando el movimiento longitudinal y lateral, creando así un movimiento elíptico que aumenta la eficacia del sistema ${ }^{11}$.

La evidencia ha demostrado una eficacia superior de la faco torsional en comparación con el desempeño general de la faco longitudinal, al reducir la cantidad de energía disipada acumulada (CDE) aplicada al ojo, lo que resulta en un tiempo de rehabilitación más corto, menor respuesta inflamatoria, tasas de complicaciones más bajas y un mejor resultado visual ${ }^{2,10,11}$.

\section{Objetivo}

El objetivo de este estudio fue comparar la eficacia global de dos plataformas de facoemulsificación, ambas con características de ultrasonido longitudinal y torsional, el sistema Active-Fluidics Centurion ${ }^{\circledR}$ y el sistema Gravity-Fluidics Infiniti ${ }^{\circledR}$ Intelligent Phaco (IP) para cataratas de grado I a IV.

\section{Métodos}

El presente estudio longitudinal, experimental y comparativo fue aprobado por la Junta de Revisión Interna del Hospital de la Asociación para Evitar la Ceguera. Todos los procedimientos cumplieron los principios de la Declaración de Helsinki. Antes de realizar los procedimientos quirúrgicos, todos los participantes aceptaron $y$ firmaron un consentimiento informado por escrito.

Incluimos pacientes con cataratas sometidos a cirugía en el Centro Visual Querétaro, Querétaro, México, desde febrero de 2015 hasta febrero de 2017.

Los pacientes reclutados fueron asignados a una de dos configuraciones quirúrgicas, fluídica activa o por gravedad. La configuración de fluídica activa comprende el sistema de visión Centurion ${ }^{\circledR}$ con fluídica activa (Active Fluidics ${ }^{\mathrm{TM}}$ ) con una punta equilibrada Intrepid de $45^{\circ} \mathrm{ABS}$ de $0.9 \mathrm{~mm}$ (Laboratorios Novartis, Basilea, Suiza). Para la configuración de fluídica por gravedad, utilizamos el sistema Infiniti ${ }^{\circledR}$ Vision y una punta Kelman de $45^{\circ}$ "mini-flared" ABS de 0.9 mm (Laboratorios Novartis, Basilea, Suiza). En ambos grupos se utilizó la manga de infusión "Ultra" de $0.9 \mathrm{~mm}$.

Las cataratas se clasificaron antes de la aleatorización con base en el Sistema estandarizado de clasificación de opacidades del cristalino III (LOCS III), en uno de cuatro grupos, específicamente, I a IV (IV es equivalente a NO5 y NO6 del LOCS III) ${ }^{12}$. 


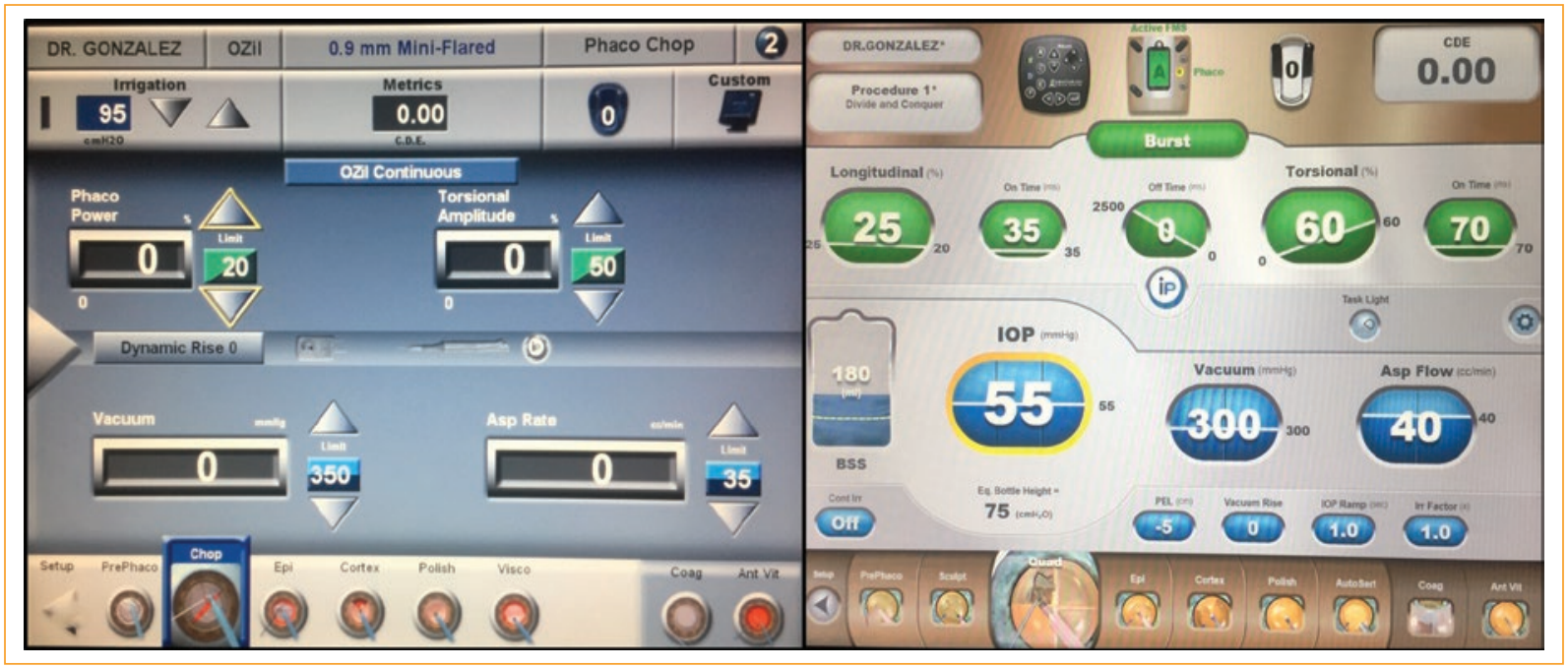

Figura 1. Pantalla de la interfaz de los dos sistemas de facoemulsificación.

\section{Pacientes}

Los criterios de inclusión clave incluyeron pacientes de 50 años de edad y mayores con opacidades del cristalino N01NC1 a NO6NC6 sometidos a cirugía de facoemulsificación e implantación de LIO. Los criterios de exclusión incluyeron patología corneal, antecedentes de uso de lentes de contacto, glaucoma, traumatismo ocular y cirugía ocular previa.

\section{Técnica quirúrgica}

Todas las cirugías fueron realizadas por un solo cirujano (RGS) utilizando una técnica de chop horizontal o vertical estándar bajo anestesia tópica. Se realizó una técnica quirúrgica de rutina con una incisión en la córnea clara de $2.4 \mathrm{~mm}$ y una capsulorrexis manual de 5.0 a $5.5 \mathrm{~mm}$ utilizando el dispositivo viscoquirúrgico oftálmico Duovisc ${ }^{\circledR}$ (hialuronato de sodio al $3 \%$; sulfato de condroitina al $4.0 \%$ con hialuronato de sodio al $1.0 \%)$. La facoemulsificación se realizó mediante la configuración quirúrgica asignada durante la aleatorización. Los parámetros de fluídica se establecieron en un límite de vacío de $350 \mathrm{mmHg}$ y una velocidad de flujo de aspiración de $40 \mathrm{ml} / \mathrm{min}$. Los parámetros del ultrasonido se fijaron en un $35 \%$ de poder lineal con un $60 \%$ de amplitud torsional. Después de la extracción de la catarata y la aspiración del material cortical, a todos los pacientes se les realizó implantación de LIO en la bolsa.

\section{Medidas de resultados}

El parámetro intraoperatorio seleccionado como la medida de resultado primaria fue la CDE, que se muestra automáticamente en la interfaz de ambos sistemas de facoemulsificación y se mide en porcentaje-segundos, como se muestra en la figura 1. Además, los datos analizados incluyeron el tiempo de aspiración y el fluido estimado; todos ellos calculados automáticamente por ambas plataformas.

La CDE se calculó utilizando la siguiente ecuación: $\mathrm{CDE}=$ media de la potencia de faco $\times$ tiempo de faco . Para el ultrasonido torsional, $\mathrm{CDE}=$ media de la amplitud torsional de la faco $\times 0.4$. La constante 0.4 se utiliza para compensar las diferencias en los parámetros de trabajo del sistema de facoemulsificación. Ambas ecuaciones están en línea con las pautas del fabricante de la unidad de faco y con estudios previos ${ }^{10,13}$.

\section{Análisis estadístico}

Las variables continuas y categóricas se muestran como medias \pm desviación estándar (DE) y porcentajes, respectivamente. Las diferencias entre las variables continuas se evaluaron mediante una prueba $t$ de Student o la prueba de Mann-Whitney según la distribución de variables. Las diferencias entre las variables categóricas se analizaron empleando la prueba exacta de Fisher. Las pruebas de ANOVA y de Kruskal-Wallis se emplearon para las comparaciones múltiples entre 
densidades de cataratas dependiendo de la distribución variable. Los valores de $p<0.05$ se consideraron estadísticamente significativos. La distribución Gaussiana se determinó utilizando la prueba de normalidad Omnibus D'Agostino-Pearson para todas las variables. El análisis estadístico se realizó utilizando el software Statistical Package for Social Sciences (SPSS) (versión 20, SPSS, Inc., Chicago, IL, EE. UU.). Además, el software Prism GraphPad (Prism Inc. versión 6.0) se empleó para las gráficas y diseños de los datos.

\section{Resultados}

Un total de 216 ojos de 216 pacientes, 122 en el grupo de fluídica activa y 94 en el grupo de fluídica por gravedad, fueron incluidos en el estudio. Los datos clínicos y demográficos se resumen en la tabla 1. Los dos grupos fueron similares en términos de edad y género; y no se evidenciaron diferencias estadísticamente significativas.

Los datos generales de CDE se presentan en la tabla 2, que muestra los valores de CDE de las cataratas con grados I a IV. En la tabla 3, se muestra una comparación de los parámetros quirúrgicos entre los grupos según el grado de densidad nuclear.

La figura 2 muestra la CDE global por densidad nuclear (LOCS III), donde se evidenciaron diferencias estadísticamente significativas para las cataratas de grado II a IV ( $p=0.031, p=0.045$ y $p=0.032$, respectivamente).

El tiempo de aspiración de acuerdo con la densidad nuclear se muestra en la figura 3 , donde se encontraron diferencias estadísticamente significativas entre los grupos para las cataratas grados II y III ( $p=0.024$ y $p=0.001$, respectivamente).

El fluido estimado utilizado se muestra en la figura 4. Se encontraron diferencias estadísticamente significativas entre las plataformas para las cataratas de grado II y III de acuerdo con la opacidad nuclear ( $p=0.001$ y $p=0.042$, respectivamente).

\section{Discusión}

La introducción del ultrasonido torsional ha contribuido significativamente a que la cirugía de catarata sea más efectiva y segura al reducir los riesgos asociados con el ultrasonido longitudinal tradicional; por lo tanto, se promueve un tiempo de rehabilitación más rápido y una mejor agudeza visual. Es ampliamente conocido que la reducción del poder total de ultrasonido durante la cirugía disminuye la pérdida de células endoteliales
Tabla 1. Datos clínicos y demográficos de los pacientes incluidos por grupo

\begin{tabular}{|c|c|c|c|}
\hline $\begin{array}{l}\text { Parámetro } \\
\text { clínico }\end{array}$ & $\begin{array}{l}\text { Fluídica } \\
\text { activa }\end{array}$ & $\begin{array}{l}\text { Fluídica por } \\
\text { gravedad }\end{array}$ & *Valor de p \\
\hline Edad (años) & $67.22 \pm 10.80$ & $62.0 \pm 12.38$ & 0.279 \\
\hline Mínimo & 57 & 58 & - \\
\hline Máximo & 91 & 92 & - \\
\hline Género (M/F) & $54 / 68$ & $43 / 51$ & - \\
\hline
\end{tabular}

Tabla 2. Valores generales de CDE, cataratas de grado I a VI según LOCS II

\begin{tabular}{|c|c|c|c|}
\hline $\begin{array}{l}\text { Densidad } \\
\text { nuclear }\end{array}$ & $\begin{array}{l}\text { Fluídica } \\
\text { activa }\end{array}$ & $\begin{array}{l}\text { Fluídica por } \\
\text { gravedad }\end{array}$ & *Valor de p \\
\hline $\begin{array}{l}\text { Grado I } \\
\text { Media } \pm D E \\
\text { Rango }\end{array}$ & $\begin{array}{c}0.78 \pm 0.13 \\
0-4.08\end{array}$ & $\begin{array}{c}0.95 \pm 1.21 \\
0-4.71\end{array}$ & 0.786 \\
\hline $\begin{array}{l}\text { Grado II } \\
\quad \text { Media } \pm \text { DE } \\
\text { Rango }\end{array}$ & $\begin{array}{c}2.25 \pm 1.43 \\
0.96-6.15\end{array}$ & $\begin{array}{l}3.61 \pm 2.3 \\
1.26-10.5\end{array}$ & 0.031 \\
\hline $\begin{array}{l}\text { Grado III } \\
\quad \text { Media } \pm \text { DE } \\
\text { Rango }\end{array}$ & $\begin{array}{c}5.39 \pm 3.15 \\
2.1-14.22\end{array}$ & $\begin{array}{l}8.07 \pm 2.3 \\
1.9-15.31\end{array}$ & 0.045 \\
\hline $\begin{array}{l}\text { Grado IV } \\
\text { Media } \pm \text { DE } \\
\text { Rango }\end{array}$ & $\begin{array}{l}12.6 \pm 0.85 \\
5.19-39.57\end{array}$ & $\begin{array}{c}16.75 \\
10.22-24.03\end{array}$ & 0.032 \\
\hline
\end{tabular}

*Se realizó una prueba t pareada para detectar diferencias entre grupos.

precipitada por el procedimiento ${ }^{9-15}$. El objetivo de nuestro estudio fue comparar la eficacia quirúrgica de dos plataformas de facoemulsificación con ultrasonido longitudinal y torsional.

Se han reconocido tres mecanismos diferentes de traumatismo inducido quirúrgicamente durante la facoemulsificación: un componente mecánico debido a la manipulación directa de los tejidos e instrumentos dentro del ojo, el poder del ultrasonido y la dinámica de la flú́dica ${ }^{16}$. El componente mecánico no ha sido de interés en estudios previos debido a los desafíos para evaluarlo y su dependencia de la curva de aprendizaje de los cirujanos. Sin embargo, al estandarizar todos los procedimientos a un solo cirujano, el componente mecánico, como factor de confusión de los análisis estadísticos presentados, no es motivo de preocupación en nuestro estudio. 
Tabla 3. Comparación de los parámetros quirúrgicos entre grupos según el grado de densidad nuclear

\begin{tabular}{|c|c|c|c|c|}
\hline Parámetro & Grado & Fluídica activa & Fluídica por gravedad & Valor de $\mathrm{p}^{*}$ \\
\hline \multirow[t]{4}{*}{ CDE (porcentaje-segundos) } & Grado I & $1.88 \pm 0.18$ & $1.31 \pm 0.21$ & 0.279 \\
\hline & Grado II & $2.72 \pm 0.46$ & $3.12 \pm 0.40$ & 0.031 \\
\hline & Grado III & $5.874 \pm 0.73$ & $7.92 \pm 0.90$ & 0.045 \\
\hline & Grado IV & $13.72 \pm 1.96$ & $18.71 \pm 2.11$ & 0.032 \\
\hline \multirow[t]{4}{*}{ TA (ms) } & Grado I & $247.4 \pm 25.18$ & $247.9 \pm 11.2$ & 0.250 \\
\hline & Grado II & $299.3 \pm 38.1$ & $333.5 \pm 43.1$ & 0.024 \\
\hline & Grado III & $263.9 \pm 39.1$ & $310.8 \pm 47.0$ & 0.001 \\
\hline & Grado IV & $312.9 \pm 31.6$ & $304.1 \pm 45.3$ & 0.575 \\
\hline \multirow[t]{4}{*}{$\mathrm{FE}(\mathrm{mL})$} & Grado I & $84.69 \pm 16.18$ & $88.1 \pm 15.4$ & 0.237 \\
\hline & Grado II & $91.1 \pm 11.8$ & $104.64 \pm 16.0$ & 0.001 \\
\hline & Grado III & $93.4 \pm 18.4$ & $105.61 \pm 22.0$ & 0.042 \\
\hline & Grado IV & $106.2 \pm 20.1$ & $110.1 \pm 25.6$ & 0.181 \\
\hline
\end{tabular}

*Se realizó una prueba t no pareada para detectar diferencias estadísticamente significativas entre los grupos.

CDE: energía disipada acumulada; TA: tiempo de aspiración; FE: fluido estimado utilizado (todo expresado en medias \pm desviación estándar).

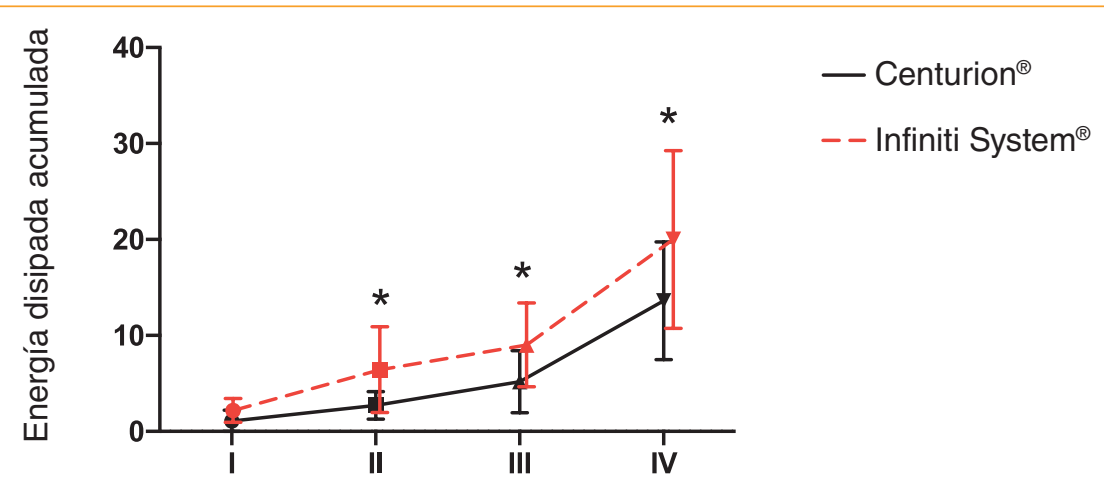

Densidad del núcleo de acuerdo a la clasificación de LOCS III, $p \leq 0.05^{*}$

Figura 2. Recuento de CDE expresada en media ( \pm DE) según la densidad nuclear (LOCS III = Sistema de clasificación de opacidades de cristalino II).

${ }^{*}$ Se realizó una prueba t no pareada para detectar diferencias estadísticamente significativas entre los grupos.

La energía disipada acumulada ha sido el principal resultado evaluado en artículos recientes que comparan diferentes tipos de sistemas de facoemulsificación ${ }^{9-11,16-18}$. Solomon, et al. encontraron una diferencia estadísticamente significativa de la CDE $(p<0.001)$ a favor de la configuración con fluídica activa en comparación con fluídica por gravedad ${ }^{17}$. De acuerdo con estos hallazgos, Oh, et al. informaron una reducción de la CDE en la configuración de fluídica activa $(p=0.017)^{18}$, y Chen, et al. demostraron una reducción del $38.2 \%$ de la CDE $(p<0.001)^{13}$.
Al estratificar nuestra población de estudio por densidad nuclear, intentamos evaluar si se podrían encontrar diferencias en la eficacia de los sistemas entre diversos grados de cataratas nucleares. Se evidenció una diferencia significativa en la CDE para las cataratas de grados II a IV $(p=0.031, p=0.045$ y $p=0.032$, respectivamente), que favorecía al grupo de fluídica activa. Pocos estudios compararon las mismas plataformas de facoemulsificación y estratificaron a su población de acuerdo con las densidades nucleares, y la 


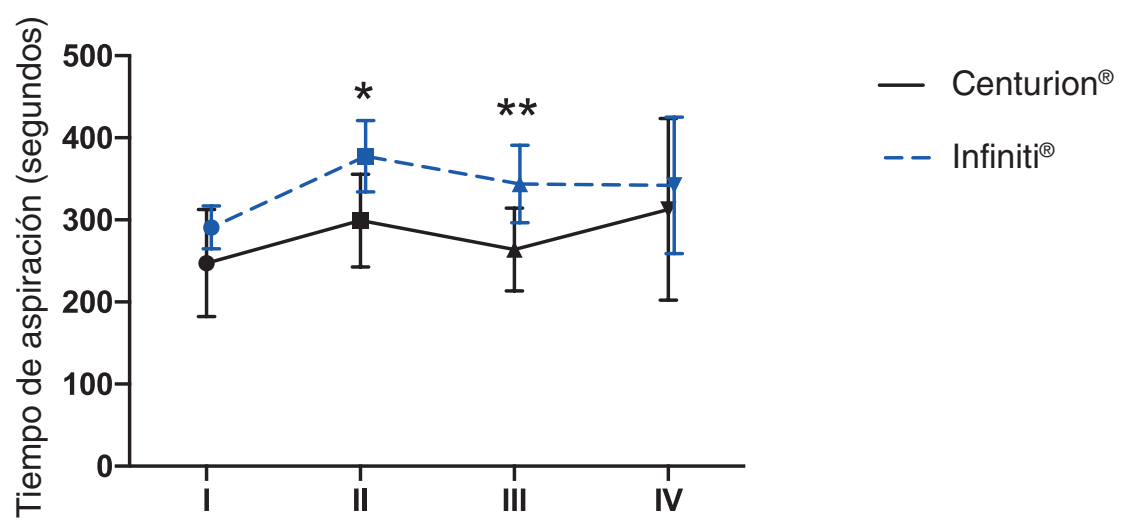

Densidad del núcleo de acuerdo a la clasificación de LOCS III,

$$
p \leq 0.05^{\star}, p \leq 0.001^{* *}
$$

Figura 3. Media $( \pm \mathrm{DE})$ del tiempo de aspiración según la densidad nuclear (LOCS III = Sistema de clasificación de opacidades de cristalino II).

* Se realizó una prueba t no pareada para detectar diferencias estadísticamente significativas entre los grupos.

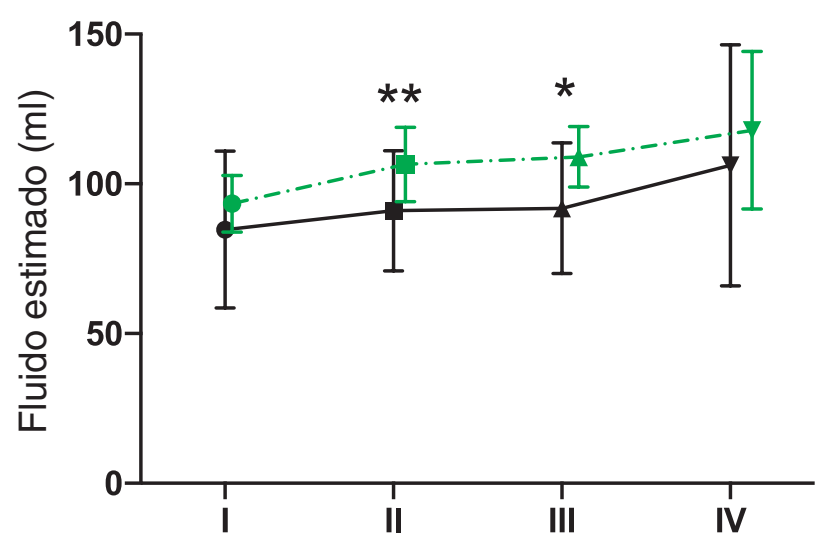

$\rightarrow$ Centurion $^{\circledR}$

- - Infiniti ${ }^{\circledR}$

Densidad del núcleo de acuerdo a la clasificación de LOCS III,

$$
p \leq 0.05^{*}, p \leq 0.001^{* *}
$$

Figura 4. Media ( \pm DE) del fluido estimado según la densidad nuclear (LOCS III = Sistema de clasificación de opacidades de cristalino II).

* Se realizó una prueba t no pareada para detectar diferencias estadísticamente significativas entre los grupos.

mayoría de ellos informaron solamente la reducción total de CDE en todo el grupo $13,16,17$.

De manera similar a nuestros hallazgos, Oh, et al. informaron una CDE más baja para cataratas de grados III y superiores $(p=0.033)$. Al tiempo que proporcionaron información muy valiosa, con una población total de 412 sujetos, incluso más grande que la de nuestro estudio, Oh, et al. no aleatorizaron a los sujetos antes de la intervención, y como consecuencia tuvieron una mayor proporción de núcleos más densos en el grupo de fluídica activa ${ }^{18}$.

En nuestro estudio, se registró un menor tiempo de aspiración para los subgrupos de cataratas de grado II y III ( $p=0.024$ y $p=0.001$, respectivamente), y menos fluido estimado ( $p=0.001$ y $p=0.042$, respectivamente) utilizando la configuración de fluídica activa. Estos resultados concuerdan con los datos informados previamente referentes únicamente a cataratas de grado II y III $^{16}$. 
De los tres resultados principales, solo la CDE fue significativamente más baja en el grupo de fluídica activa para cataratas de grado IV $(p=0.032)$. El tiempo de aspiración y el fluido estimado fueron similares en ambos grupos para las cataratas de grado I y IV. El único estrato en el que no se encontraron diferencias de CDE fue el de las cataratas de grado I. Si bien no se encontró una correlación positiva entre la dureza nuclear y la eficacia del sistema en general, nuestros hallazgos sugieren que la configuración de fluídica activa tiene un mejor desempeño general para cataratas de grado II y superior.

Se deben observar algunas limitaciones de nuestro estudio. Empleamos diferentes puntas de faco en cada plataforma, lo que puede influir en la eficacia quirúrgica general de ambos sistemas. Los datos publicados recientemente por Khokhar, et al. sugieren que la nueva punta equilibrada realizó la facoemulsificación de manera más efectiva en comparación con la punta convencional de 45 grados, especialmente en cataratas duras, que requieren mayor energía y un tiempo de facoemulsificación prolongado ${ }^{19}$. Creemos que tanto el tipo de sistema de facoemulsificación empleado como la punta de faco utilizada pueden desempeñar un papel en la eficacia general de los sistemas.

\section{Agradecimientos}

Agradecemos la invaluable contribución a este estudio de investigación de los siguientes médicos: Alberto Ansart Berges, Martín Ayala Flores, Francisco Chávez-Méndez, Patricia Culebro Solano, José Feregrino Ortiz, Martín Gallegos Duarte, Vinicio García Solís, Elizabeth Gómez-Pimienta, Roberto González Origel, Miguel Huerta García, Jorge A. León Carrera, Rocío Meré Alcocer, Lorley Miller Eagan, Jorge Pacheco Padrón, Jaime Palacios Alcocer, Lourdes Páramo Figueroa, Judith Peñaflor Siller, Nicolás Perea Ortega, Hector Randolph Rodríguez, Manuel Sáenz-de-Viteri Siso, Manuel Sáenz-de-Viteri Vázquez, Roberto Varela Gress y Patricia Vázquez Rojas.

\section{Conflicto de intereses}

Los autores no tienen ningún interés económico, de propiedad o financiero que revelar en la publicación de este documento.

\section{Financiamiento}

No se recibió apoyo financiero para realizar este estudio.

\section{Responsabilidades éticas}

Protección de personas y animales. Los autores declaran que para esta investigación no se han realizado experimentos en seres humanos ni en animales.

Confidencialidad de los datos. Los autores declaran que han seguido los protocolos de su centro de trabajo sobre la publicación de datos de pacientes.

Derecho a la privacidad y consentimiento informado. Los autores han obtenido el consentimiento informado de los pacientes y/o sujetos referidos en el artículo. Este documento obra en poder del autor de correspondencia.

\section{Bibliografía}

1. Lundström M, Barry P, Henry Y, Rosen P, Stenevi U. Evidence-based guidelines for cataract surgery: guidelines based on data in the European Registry of Quality Outcomes for Cataract and Refractive Surgery database. J Cataract Refract Surg. 2012;38(6):1086-93.

2. Reuschel A, Bogatsch H, Barth T, Wiedemann R. Comparison of endothelial changes and power settings between torsional and longitudinal phacoemulsification. J Cataract Refract Surg. 2010;36(11):1855-61.

3. Pascolini D, Mariotti SP. Global estimates of visual impairment: $2010 . \mathrm{Br}$ J Ophthalmol. 2012;96:614-8.

4. Riaz Y, de Silva SR, Evans JR. Manual small incision cataract surgery (MSICS) with posterior chamber intraocular lens versus phacoemulsification with posterior chamber intraocular lens for age-related cataract. Cochrane Database Syst Rev. 2013;(10): CD008813.

5. Spalton D, Koch D. The constant evolution of cataract surgery. BMJ. 2000;321:1304.

6. Lansingh VC, Carter MJ, Martens M. Global cost-effectiveness of cataract surgery. Ophthalmology. 2007;114:1670-8.

7. Abell RG, Vote BJ. Cost-Effectiveness of Femtosecond Laser-Assisted Cataract Surgery versus Phacoemulsification Cataract Surgery. Ophthalmology. 2014;121:10-6.

8. McAlinden C, Wang Q, Pesudovs K, Yang X, Bao F, Yu A, et al. Axial length measurement failure rates with the IOLMaster and Lenstar LS 900 in eyes with cataract. PLoS One. 2015;10(6):e0128929.

9. Christakis PG, Braga-Mele RM. Intraoperative performance and postoperative outcome comparison of longitudinal, torsional, and transversal phacoemulsification machines. J Cataract Refract Surg. 2012;38:234-41.

10. Rẹkas M, Montés-Micó R, Krix-Jachym K, Kluś A, Stankiewicz A, Ferrer-Blasco T. Comparison of torsional and longitudinal modes using phacoemulsification parameters. J Cataract Refract Surg. 2009; 35(10):1719-24.

11. Assaf A, Roshdy MM. Comparative analysis of corneal morphological changes after transversal and torsional phacoemulsification through $2.2 \mathrm{~mm}$ corneal incision. Clin Ophthalmol. 2013;7:55-61.

12. Chylack LT, Wolfe JK, Singer DM, Leske MC, Bullimore MA, Bailey IL, et al. The lens opacities classification system III. Arch Ophthalmol. 1993; $111(6): 831-6$.

13. Chen M, Anderson E, Hill G, Chen JJ, Patrianakos T. Comparison of cumulative dissipated energy between the Infiniti and Centurion phacoemulsification systems. Clin Ophthalmol. 2015;9:1367-72.

14. Sorrentino FS, Matteini S, Imburgia A, Bonifazzi C, Sebastiani A, Parmeggiani F. Torsional phacoemulsification: A pilot study to revise the "harm scale" evaluating the endothelial damage and the visual acuity after cataract surgery. PLoS One. 2017;12(10):e0186975.

15. Venkatesh R, Van Landingham SW, Khodifad AM, Haripriya A, Thiel CL, Ramulu $P$, et al. Carbon footprint and cost-effectiveness of cataract surgery. Curr Opin Ophthalmol. 2016 Jan 1;27(1):82-8.

16. Gonzalez-Salinas R, Garza-Leon M, Saenz-de-Viteri M, Solis-S JC, Gulias-Cañizo R, Quiroz-Mercado H. Comparison of cumulative dissipated energy delivered by active-fluidic pressure control phacoemulsification system versus gravity-fluidics. Int Ophthalmol. 2017;1-7.

17. Solomon KD, Lorente R, Fanney D, Cionni RJ. Clinical study using a new phacoemulsification system with surgical intraocular pressure control. J Cataract Refract Surg. 2016;42:542-9.

18. Oh LJ, Nguyen CL, Wong E, Wang SS, Francis IC. Prospective study of Centurion ${ }^{\circledR}$ versus Infiniti ${ }^{\circledR}$ phacoemulsification systems: surgical and visual outcomes. Int J Ophthalmol. 2017;10(11):1698.

19. Khokhar S, Aron N, Sen S, Pillay G, Agarwal E. Effect of balanced phacoemulsification tip on the outcomes of torsional phacoemulsification using an active-fluidics system. J Cataract Refract Surg. 2017;43(1):22-8.

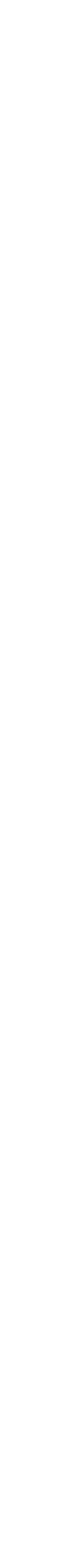

